\title{
EDUCACIÓN Y CONTEXTOS VULNERABLES: RETOS Y RECURSOS PARA LA PREVENCIÓN DEL CONSUMO DE DROGAS Y OTRAS ADICCIONES
}

\author{
Ángel Manuel Turbi Pinazo \\ Universidad Católica de Valencia \\ angelmanuel.turbi@ucv.es
}

Fecha de Recepción: 8 Marzo 2019

Fecha de Admisión: 30 Abril 2019

\section{RESUMEN}

Desde el contexto educativo del Proyecto Scholas Occurrentes, y concretando, desde la Cátedra de la Universidad Católica de Valencia, consideramos que hemos de fomentar y facilitar un cambio en la educación; por lo que dentro de este ámbito hemos reflexionar sobre la necesidad de una intervención con perspectiva socioantropológica para llegar a una intervención educativa, desde la evidencia científica y la aportación académica-universitaria.

En este contexto no podemos obviar la realidad de las conductas adictivas, priorizando y definiendo la vulnerabilidad contextual, para desde ahí, alcanzar la era digital/virtual en la que nuestros/as adolescentes se encuentran inmersos, tanto desde el ámbito educativo y social, emergiendo nuevas conductas adictivas, y por "ende" nuevas violencias.

Contextualizaremos los conceptos de educación y vulnerabilidad, llegando al análisis de los factores de riesgo y protección que facilitan o predisponen a nuestros adolescentes a la evolución 0 cronificación de una conducta adictiva.

Dentro del ámbito educativo-preventivo, señalamos niveles y ámbitos (aceptando una parte del fracaso en la intervención preventiva que se ha realizado en la últimas décadas), revisando nuestras acciones, proponiendo nuevos enfoques y técnicas.

No podemos eludir una nueva realidad: la era digital/virtual existente, generando necesidad de educar al respecto a los adolescentes, considerados nativos digitales; el desarrollo de las TIC (Tecnologías de la Información y Comunicación) y otras tecnologías emergentes, realidad cambiante, con sus ventajas y sus riesgos, junto a la aparición de las tecnoadicciones, y sus consecuencias: "nuevas violencias desde las redes sociales", haciendo especial mención al Ciberbullying, problemática que desde Scholas Occurrentes, preocupa y se ocupa sensibilizando y actuando en un contexto educativo, formativo incluyendo al profesorado.

Palabras clave: educación; vulnerabilidad; adicciones; tecnoadicciones; cyberbullying 


\section{ABSTRACT}

Education and vulnerable contexts: challenges and resources for the prevention of drug consumption and other addictions. From the educational context of the Scholas Occurrentes Project, and concretely, from the Chair of the Catholic University of Valencia, we consider that we must encourage and facilitate a change in education; Therefore, within this area we have to reflect on the need for an intervention with a socioanthropological perspective to arrive at an educational intervention, based on scientific evidence and the academic-university contribution.

In this context we can not ignore the reality of addictive behaviors, prioritizing and defining contextual vulnerability, from there, reaching the digital / virtual era in which our adolescents are immersed, both from the educational and social, emerging new addictive behaviors, and by "ende" new violence.

Contextualize the concepts of education and vulnerability, reaching the analysis of risk factors and protection that facilitate or predispose our adolescents to the evolution or chronification of an addictive behavior.

Within the educational-preventive field, we indicate levels and scopes (accepting a part of the failure in the preventive intervention that has been made in the last decades), reviewing our actions, proposing new approaches and techniques.

We can not avoid a new reality: the existing digital / virtual era, generating a need to educate adolescents, considered digital natives; the development of ICT (Information and Communication Technologies) and other emerging technologies, a changing reality, with its advantages and risks, together with the appearance of techno addictions, and its consequences: "new violence from social networks", making special mention to the Cyberbullying, problematic that from Scholas Occurrentes, worries and takes care sensitizing and acting in an educative, formative context including the teaching staff.

Keywords: education; vulnerability; addictions; techno addictions; cyberbullying

\section{INTRODUCCIÓN: EDUCACIÓN Y VULNERABILIDAD}

La pretensión de las siguientes líneas no es más que considerar a la "persona" receptora de la educación en lo más alto de dicha acción, "educar" para y por ella, teniendo como fin último potenciar al máximo sus posibilidades, atendiendo a su realidad y contexto, generando un estado de Bienestar, y siguiendo a la OMS y su definición de salud (Declaración de Alma Atá, 1977): la salud es un estado completo e integral de bienestar físico, psicológico y social de la persona. Si nuestra misión educativa la encaminamos en este sentido, no podemos obviar el contexto en que desarrollamos la misma, que en ocasiones es de vulnerabilidad.

\section{FACTORES DE RIESGO Y PROTECCIÓN}

Entendemos como factor de riesgo "un atributo y/o característica individual, condición situacional y/o contexto ambiental que incrementa la probabilidad del uso y/o abuso de drogas (inicio) 0 una transición en el nivel de implicación con las mismas (mantenimiento)" (Clayton, 1992, p. 15). A su vez, consideramos un factor de protección "un atributo o característica individual, condición situacional y/o contexto ambiental que inhibe, reduce 0 atenúa la probabilidad del uso y/o abuso de drogas o la transición en el nivel de implicación con las mismas" (Clayton, 1992, p. 16).

Centrándonos en los factores de riesgo y por sistematizar los mismos, consideramos la clasificación de Hawkins et al. (1992), como guía sistemática, si bien, no la queremos considerar como axioma único, sino como marco, entre otras clasificaciones como Petterson et al. (1992) y la de Moncada (1997). Resaltamos por supuesto, y teniendo en cuenta la realidad de nuestra interven- 
ción, que la persona es única junto a sus circunstancias, por lo que dicha intervención hay que adecuarla al individuo, sin caer en el error de aplicar meras teorías o programas.

\section{Tabla 1}

Factores de riesgo para el abuso de sustancias psicoactivas

\section{Genéticos}

Hijos de consumidores de sustancias psicoactivas

\section{Constitucionales}

Uso temprano de drogas (antes de los 15 años)

Dolor o enfermedad crónica

Factores fisiológicos

\section{Psicológicos}

Problemas de salud mental

Abuso físico, sexual o emocional

\section{Socioculturales:}

\section{Familiares}

Uso de drogas por parte de los padres y actitudes positivas hacia el uso de drogas

Divorcio o separación de los padres

Problemas de manejo de la familia

Bajas expectativas para los niños o para el éxito

\section{Iguales}

Amigos que usan drogas

Actitudes favorables hacia el uso de drogas

Conducta antisocial o delincuente temprana

\section{Escuela}

Carencia de cumplimiento de la política escolar

Poca dedicación a la escuela

Fracaso escolar o abandono de la escuela

Dificultades en el paso de uno a otro curso

\section{Comunitarios}

Leyes y normas de la comunidad favorables hacia el uso de drogas

Carencia de una adecuada vinculación social

Deprivación económica y social

Disponibilidad de drogas (incluyendo alcohol y tabaco)

Fuente: elaboración propia adaptado de Hawkins et al. (1992). 


\section{EDUCACIÓN Y CONTEXTOS VULNERABLES: RETOS Y RECURSOS PARA LA PREVENCIÓN DEL CONSUMO DE DROGAS Y OTRAS ADICCIONES}

Queremos resaltar algunos de estos factores de protección, que encajan perfectamente en la temática que nos ocupa en este manual. En primer lugar, señalaremos algunos de los considerados individuales:

- La religiosidad desde un orden moral y de habilidades sociales, como factor de protección individual (Pollard et al. 1997). Tal y como señalan Chavez, Oetting y Swaim (1994), asistir a la iglesia, sostener una afiliación religiosa, aportando ambas variables un apoyo social, un significado ante la búsqueda de identidad y un apego social en entornos grupales alejados del consumo (Félix-Ortiz y Newcomb, 1999), junto a un buen rendimiento académico y poseer un proyecto de vida con miras al futuro, son un factor de protección ante el consumo de drogas.

- Otro factor de protección es la inteligencia (Fergusson y Lynskey, 1996; Jessor, 1991), si bien no lo consideramos uno de los más significativos o relevantes.

- La resiliencia: concepto que en ocasiones ha sido abusado (y sigue siendo) en la literatura científica. Existen numerosas definiciones del concepto si bien, adoptaremos la de Garmezy (1991), considerando la resiliencia como "la capacidad para recuperarse y mantener una conducta adaptativa que puede seguir a una retirada o incapacidad inicial después de iniciarse un evento estresante", desde aquí es donde entendemos este concepto como un posible factor de protección frente al consumo de drogas y dando una aplicación específica al tema que nos ocupa podemos definir la resiliencia por las siguientes capacidades presentes en algunos de nuestro niños/as (Kumpfer et al., 1998).Siguiendo a Rutter (1985), la resiliencia conlleva varios componentes: autoestima y autoconfianza, expectativas de autoeficacia y habilidades de afrontamiento ante un cambio con la capacidad de adaptación a éstos, junto a habilidades que permitan la solución de problemas sociales. Por lo que ante el riesgo de aparición de conductas adictivas la persona sería inmune ante dicho consumo (Becoña, 2002).

Podríamos seguir y ampliar el concepto como factor de protección si bien, dejaremos una cuestión para la reflexión del lector ¿la resiliencia se aprende o tiene componente innato 0 desarrollado por las vivencias?, concluimos con uno de los autores que contribuyeron a la difusión del concepto, la resiliencia lleva que "ninguna herida sea un destino" (Cyrulnik, 2002).

Tras los factores de protección señalados anteriormente, relativos a lo individual, no queremos pasar por alto los relacionados con la educación, en concreto al ámbito escolar.

- Un escuela de calidad, donde todos los componentes de la misma, especialmente los docentes contribuyen a un funcionamiento adecuado que respetando los periodos evolutivos de los alumnos/as favorezcan un desarrollo integral de los mismos/as (Jessor, 1991).

No podemos abandonar dicho apartado sin hacer una breve referencia al mundo de los valores, de la ética y moral, aquello que nos permite discernir entre el bien y el mal (Tevez, 2006). La falta de valores-éticos están contribuyendo a la creación de nuevos contextos vulnerables.

\section{NUEVOS PLANTEAMIENTOS EN PREVENCIÓN}

Tradicionalmente y desde una perspectiva clásica, los programas preventivos se consideraban primarios, secundarios y terciarios, si bien tal y como ya señaló hace unos años Becoña (2002), éstos en la actualidad se clasifican según su nivel de actuación, considerando el colectivo objeto de la intervención; en primer lugar, haremos un repaso histórico siguiendo a Becoña (2001):

- Los primeros modelos son los llamados modelos racionales e informativos.

- Modelo de influencias sociales o psicosociales (Perry y Kelder, 1992).

- Modelo de habilidades generales (Life Skills Trainning, LST) elaborado por Botvin (1995).

Tras dicha revisión describimos brevemente los actuales niveles de prevención aceptados en la prevención de las adicciones (Becoña, 2002; Gordon, 1987; Turbi, 2013): 
- La prevención universal sería aquella que puede dirigirse a todos los adolescentes, beneficiándolos a todos por igual. Son perspectivas amplias, menos intensas y menos costosas que otras que se orientan a conseguir cambios de comportamiento duraderos. Se incluirían en estos programas aquellos programas preventivos escolares que tratan de fomentar habilidades y clarificar valores.

- La prevención selectiva es aquella que se dirige a un subgrupo de adolescentes que tienen un riesgo de presentar la conducta problema mayor que el promedio de los adolescentes. Se dirigen a grupos de riesgo.

- La prevención indicada es más intensiva y más costosa. Se dirige a un subgrupo concreto de la comunidad que ya tienen problemas de comportamiento, o que presentan la conducta problema, se dirigen, por tanto, a individuos de alto riesgo.

Completamos la clasificación anterior con un nivel, poco estudiado y difundido en el campo que nos ocupa, si bien, más conocido en él la disciplina de la Educación Social, e interesante para conocer y tener en cuenta:

- Prevención determinada, dirigida a grupos/colectivos de consumidores tras un diagnóstico de drogodependencia y otros trastornos de salud relacionados. (Diputación de Barcelona, 2013).

Ya metidos en materia y con la intencionalidad de plasmar y actualizar al máximo conceptos y niveles en la materia de prevención, un nivel, que en la actualidad se está contemplando, mayormente relacionado con las políticas públicas es el de Prevención Ambiental, aún poco conocido por los técnicos del sector si bien, de suma importancia para desarrollar nuevas estrategias y políticas preventivas, que complementan las anteriormente citadas y unifican esfuerzos ante dicha problemática, reduciendo e interviniendo, como no, en contextos y momentos de vulnerabilidad; definiremos este nivel de prevención como las estrategias que alteran las normas y los ambientes físicos, sociales y económicos sin basarse en persuasión (Burkhart, 2011).

Este nivel o nueva esfera de intervención en el ámbito de la prevención conlleva importantes matices e implicaciones en el campo, ya que nos hace ver la importancia de desarrollar estrategias que actúan de forma directa en el ambiente, en el cual se dan los consumos de sustancias y a su vez las normas de nuestros y nuestras adolescentes y jóvenes se configuran y mantienen.

Cada vez más, dichas actuaciones ambientales están cobrando mayor importancia, principalmente en el Norte de Europa destacando en tres áreas: a nivel macro en forma de políticas de control de alcohol y tabaco, en estrategias de mejorar el clima en centros educativos y en políticas locales/municipales para ambientes de diversión (Burkhart, 2013).

Un paso más; una reingeniería de la prevención, nuevas perspectivas

Considerando al profesor Salazar y su equipo como promotor de uno de estos modelos, (Salazar y Bustos, 2002; Salazar y Williamson, 2015), describimos su propuesta: un modelo de prevención moderno en relación a la prevención de las drogodependencias, identificando, los Niveles de Prevención ISA, correspondiendo las siglas a lo siguiente (véase Figura 1):

a) Información, (I). Definir los elementos y conocimientos básicos que se han de tener sobre la problemática de la violencia. Esta información será la base de los siguientes niveles.

b) Sensibilización, (S). Capacidad de los sujetos para adquirir una disposición afectiva sobre la temática.

c) Actitud, (A). Disposición a comportarse u obrar de una manera favorable, quedando ésta testimoniada en el grado de compromiso como futuro agente preventivo. 
Fig. 1. Modelo ISA

Fuente: elaboración propia a partir de Salazar y Williamson (2015)

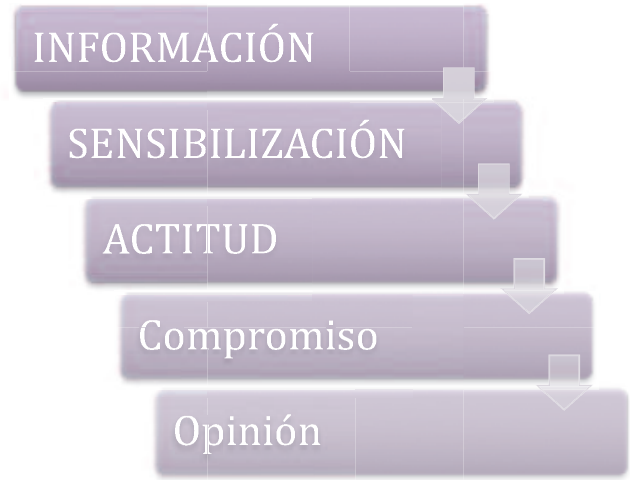

Otro de estos enfoques, que hemos considerado describir por su desconocimiento y escasa difusión, si bien ya es adoptado por diversos planes municipales en nuestro país, es el Enfoque IVAC (FAD, 2011) (véase Figura 2.):

Fig. 2. Enfoque IVAC

Fuente: elaboración propia a partir de FAD (2011)

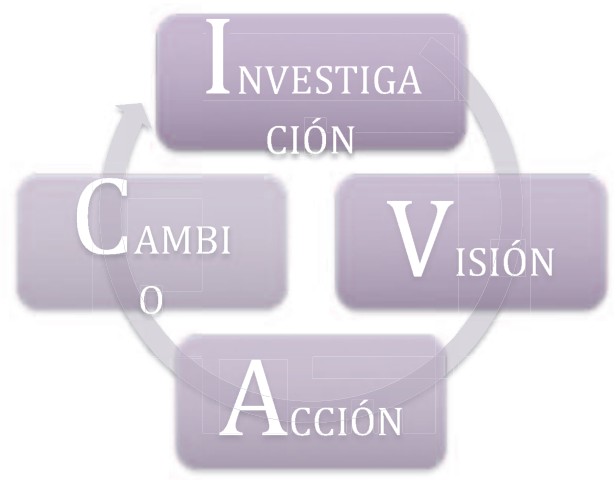

Este enfoque, muy acorde con el Modelo ISA descrito anteriormente, se basa en la siguiente metodología:

Investigación, (I). En esta primera fase, tanto los niños/as como adolescentes dependiendo del colectivo de la intervención, investigarán, por supuesto guiados por el equipo profesional, sobre la problemática a tratar, desde un punto de vista social, psicológico, cultural, salud...

Visión, (V). Tras la fase previa de investigación, es decir, de la toma de contacto y adquisición de información junto a conocimientos básicos de la temática (información que han adquirido de forma autónoma y no dada/impartida por "expertos", tal y como de forma tradicional se ha ido realizando en prevención), propondrán alternativas de conducta y soluciones ante la variable que se ha considerado relevante minimizar y prevenir, el consumo. 
Acción, (A). Es aquí, tal y como indica el nombre de dicha fase, donde los propios usuarios/alumnos, diseñan y elaboran las estrategias, que consideran tras el análisis y estudio del problema, como posibles acciones preventivas, con la intencionalidad de mejorar la calidad de vida, aumentando el bienestar tanto de ellos/as, como de sus iguales, siendo extensible a otros colectivos, es decir, pretendiendo subsanar los factores de riesgo, la vulnerabilidad, desde su propia visión.

Cambio, (C). De este modo, con metodología experiencial, se pretende una mayor interiorización de las alternativas saludables, provocando un cambio actitudinal y conductual.

\section{EL DEPORTE COMO HERRAMIENTA DE PREVENCIÓN}

Tal y como está trabajando Scholas Occurrentes en Scholas Deporte, el deporte es una estrategia que va más allá de la herramienta, que tiene como objetivo formar y concienciar en valores a niños y jóvenes por medio de esta actividad, actuando en contextos de vulnerabilidad e involucrarlos con la realidad de sus comunidades. El deporte es útil para fomentar la integración social y la cultura del encuentro.

La actividad física, al igual que la práctica deportiva, planteada como hábito saludable y actividad educativa (sin obviar, que una obsesión o excesiva competitividad pueden tener consecuencias negativas), conlleva, tanto beneficios físicos como psicológicos, que nos permiten hacer un uso preventivo potente, fomentando los factores de protección y a su vez minimizando los de riesgo ante el consumo de drogas.

En contextos vulnerables, considerando a adolescentes y los riesgos que conlleva dicho periodo evolutivo, la práctica deportiva, entendida como actividad educativa permite una mejor organización y ocupación del tiempo libre/ocio, una inserción/integración comunitaria, adoptando normas sociales, estrategias de resolución de conflictos y la interiorización de valores como el respeto, compañerismo, trabajo en equipo..., así como por ejemplo, la adquisición de autodisciplina entre otras actitudes, todas ellas consideradas factores de protección que pueden alejar a nuestro jóvenes de estilos de vida favorecedores del consumo de drogas (Hernández, Pastor y Gil, 2016).

\section{LA ERA DIGITAL: NUEVAS ADICCIONES}

Comencemos describiendo la realidad española desde la Encuesta sobre Equipamiento y Uso de Tecnologías de Información y Comunicación en los Hogares (Instituto Nacional de Estadística, 2016).

Tabla 2

Porcentaje de usuarios de TIC por sexo y edad, 2016

Fuente: INE, 2016

\begin{tabular}{|c|c|c|c|}
\hline & Uso de ordenador & Uso de Intemet & $\begin{array}{l}\text { Disposición de } \\
\text { móvil }\end{array}$ \\
\hline Total & 94,9 & 95,2 & 69,8 \\
\hline \multicolumn{4}{|l|}{ Sexo } \\
\hline Hombres & $\overline{95,6}$ & $\overline{95,7}$ & 68,8 \\
\hline Mujeres & 94,2 & 94,7 & 70,9 \\
\hline \multicolumn{4}{|l|}{ Edad } \\
\hline 10 & 92,6 & 90,6 & 25,4 \\
\hline 11 & 92,6 & 93,1 & 50,9 \\
\hline 12 & 94,8 & 95,9 & 72,7 \\
\hline 13 & 96,7 & 95,4 & 86,0 \\
\hline 14 & 95,7 & 98,2 & 90,1 \\
\hline 15 & 97,1 & 98,0 & 93,9 \\
\hline
\end{tabular}



Y OTRAS ADICCIONES

Como se puede comprobar en la Tabla 2, prácticamente el 100\% de nuestros y nuestras adolescentes, con la edad de 15 años disponen de algún dispositivo con acceso a internet, es decir, la era digital ha irrumpido en nuestras vidas provocado por un vertiginoso desarrollo de la Tecnología de la Información y Comunicación (TIC), por lo que es de suma importancia conocer este entorno y la aparición de los nuevos fenómenos relacionados con el uso de las TIC y sus redes sociales. Con la aparición de este contexto novedoso, hemos de desarrollar nuevas estrategias preventivas y de actuación, ¿reciben nuestros jóvenes una educación al respecto?, ¿se hace un uso responsable?, ¿está el sistema educativo preparado para ello?

Nos encontramos ante los nativos digitales, individuos que han crecido inmersos en la tecnología digital, dicho término lo acuñó Prensky (2001), diferenciándolos de los inmigrantes digitales, personas que han llegado más tarde a las TIC. Hoy por hoy podemos decir que nuestros adolescentes y jóvenes son los expertos tecnológicos. Como indican García, Portillo, Romo y Benito (2007), los nativos digitales son una generación que ha crecido inmersa en las tecnologías, desarrollándose entre equipos informáticos, videoconsolas y todo tipo de artilugios digitales, convirtiendo los teléfonos móviles en smartphones, los videojuegos en juegos on-line, el uso de internet en todo un mundo virtual, la mensajería instantánea en un creciente aluvión de aplicaciones con acceso directo desde cualquier momento y espacio; la tecnología es parte integral de sus vidas, donde las herramientas tecnológicas ocupan un lugar central, dependiendo de ellas para todo tipo de cuestiones cotidianas, como estudiar, relacionarse, comprar, informarse 0 divertirse.

Estos nativos digitales según García, Portillo, Romo y Benito (2007) presentan unas características concretas, entre ellas resaltamos:

Nacieron en la era digital.

Son usuarios permanentes de las tecnologías.

Su característica principal es sin duda su tecnofilia.

Sienten atracción por todo lo relacionado con las tecnologías.

Con las TIC satisfacen sus necesidades de entretenimiento, diversión, comunicación, información e incluso de formación.

Enfocan su trabajo, el aprendizaje y los juegos de nuevas formas: absorben rápidamente la información multimedia de imágenes y vídeos, igual o mejor que si fuera texto.

Consumen datos simultáneamente de múltiples fuentes; esperan respuestas instantáneas; permanecen comunicados permanentemente y crean también sus propios contenidos.

Hacen varias cosas al mismo tiempo: son multitarea y multipantalla.

Afrontan distintos canales de comunicación simultáneos, prefiriendo los formatos gráficos a los textuales.

Utilizan el acceso hipertextual en vez del lineal.

Funcionan mejor trabajando en red.

Y prefieren los juegos al trabajo serio.

Habría que analizar diversas causas de esta realidad; en diversos estudios realizados a través de encuestas a población escolar (Turbi y López, 2010). Los jóvenes han cambiado el estilo de vida, conforme a la evolución de las TIC (Bueno, 2009). De este cambio de estilo de vida inferimos otra característica de nuestros niños/as y jóvenes: para los nativos digitales existen dos realidades: la física y la virtual, para el inmigrante digital, (mayoritariamente el adulto) la realidad virtual no es real, la entiende como un entretenimiento, un juego, pero no como un estilo de vida, como una interacción con el medio.

Siguiendo en la misma línea, hagamos un repaso de estas, adicciones tecnológicas actualizadas (Turbi, 2009, 2013, 2017): 
Las principales tecnoadicciones son:

Adicción a los videojuegos.

Adicción al teléfono móvil. (en la actualidad hablaríamos de smartphone)

Adicción a Internet o Ciberadicción.

Modalidades:

Ciberjuego adictivo. (incluyendo conductas compulsivas en la red). Aquí, hemos de señalar y resaltar dos vertientes: el ciberjuego tipo videoconsola, en la actualidad siendo mayoritario el ciberjuego on-line con un potencial mayor adictivo frente al off-line; y el juego tipo casino on-line, siendo la nueva ludopatía, donde incluimos las apuestas deportivas, siendo la puerta de entrada a esta nueva ludopatía, ya presente en nuestros adolescentes y jóvenes en España.

En esta última modalidad y desde un punto de vista psiquiátrico/psicológico, la adicción al juego, según la APA (American Psychiatric Association) plasmado en su DSM, (Manual Estadístico de los Trastornos Mentales), desde su penúltima edición, DSM-IV-TR, el juego patológico se consideraba como trastorno del control de los impulsos, si bien, y ya en su última edición, DSM-5 (APA, 2013), vigente en la actualidad, el juego patológico es considerado en la actualidad como trastorno adictivo.

Cibercompra adictiva. (incluyendo conductas compulsivas en la red, como subastas).

Cibertrabajo adictivo (con consecuencias como el denominado "blurring").

Cibersexo adictivo, tanto el consumo de pornografía como prácticas cibersexuales, realidad que ha conllevado nuevas violencias en este ámbito.

Cibercomunicación adictiva (ciber-relaciones).

Ciberadicción a la información (vagabundos en la red)

\section{LA ERA DIGITAL: NUEVAS VIOLENCIAS}

El que cada vez vaya en aumento el uso de las TIC s, y dentro de ellas el aumento del uso de las redes sociales, hace que la realidad virtual de una persona crezca, por lo que se incorporan a esta realidad virtual actividades que eran propias de la interacción física. Es más, la violencia de la realidad física se ha llevado a las redes sociales, y la violencia en las redes sociales se lleva a la realidad física, con todo han aparecido nuevas formas de violencia.

En esta ocasión nos centraremos en la problemática actual del ciberbullying, una nueva forma de violencia, cada vez más extendida tras el auge de las redes sociales. Se puede denominar también ciberacoso. Siguiendo la definición del INTECO (2009: 3), Instituto Nacional de Tecnología de la Comunicación, esta conducta se define como acoso entre iguales en el entorno TIC, que incluyen actuaciones de chantaje, vejaciones e insultos de niños a otros niños (o adolescentes). En una definición más exhaustiva, se puede decir que el ciberbullying supone difusión de información lesiva 0 difamatoria en formato electrónico a través de medios de comunicación como el correo electrónico, mensajería instantánea, las redes sociales, la mensajería de texto a través de teléfonos o dispositivos móviles o la publicación de vídeos y fotografías en plataformas electrónicas de difusión de contenidos. Normalmente el acosado/a-acosador/a, suelen ser compañeros de estudios y conocerse en la vida física.

Según el Observatorio de la seguridad de la información del INTECO, en el Estudio sobre hábitos de seguridad en el uso de las TIC por niños y adolescentes y e-confianza de sus padres (2009), elaborado a partir de encuestas a menores entre 6 y 16 años y sus padres 0 tutores, nos indican que el 5,9\% afirman haber sido víctimas de ciberbullying y un 2,9\% reconoce haber sido acosador.

Haremos especial incidencia dentro de esta ciberviolencia: al informe publicado por la OMS en marzo de 2016, el cual nos alerta de la incidencia de depresión, autolesiones e incluso suicidio, por 


\section{EDUCACIÓN Y CONTEXTOS VULNERABLES: RETOS Y RECURSOS PARA LA PREVENCIÓN DEL CONSUMO DE DROGAS Y OTRAS ADICCIONES}

este motivo entre los más jóvenes, encontrándose España en el séptimo puesto del ranking de ciberacoso, (entre 42 países evaluados), con la aparición de una tendencia novedosa, "bully victim". Con todo se pretende desarrollar estrategias de intervención acordes, tanto en las vertientes de prevención y tratamiento; es más, existen evidencias de entre sufrir bullying y la aparición de síntomas psicóticos posteriores (Valmaggia et al, 2015).

No podemos dejar de nombrar tal y como describen de forma exhaustiva a nivel legislativo Pérez Vallejo y Pérez Ferrer (2016) cuestiones legislativas, siendo esta nueva forma de violencia susceptible de delito penal según la Legislación española en el Código penal como delito de acoso permanente tras la reforma de la LO 1/2015, de 30 de marzo.

\section{REFLEXIONES FINALES}

Con todo, las palabras no dejan de ser una invitación a la reflexión, a focalizar una mirada amplia, a lo cercano y a lo lejano, a lo vulnerable, a las ya conocidas problemáticas como las adicciones que dificultan el desarrollo integral de nuestros jóvenes, a las nuevas caras de las mismas como son las tecnoadicciones presentes en esta nueva era y la aparición de esas nuevas violencias, todo con el fin de motivar a un cambio educativo.

Sin más..., y como no podía ser de otra forma, cerramos estas páginas con esas palabras del Papa Francisco "no se puede cambiar el mundo si no se cambia la educación" y transmitiendo esperanza a todos y todas las personas que estamos implicadas en este cambio y a las que podemos alcanzar: OTRA EDUCACIÓN ES POSIBLE.

\section{REFERENCIAS BIBLIOGRÁFICAS}

American Psychiatric Association. (2014). Manual diagnóstico y estadístico de los trastornos mentales DSM-5. Madrid: Editorial Médica Panamericana.

Becoña Iglesias, Elisardo (2001). Bases teóricas que sustentan los programas de Prevención de drogas. Madrid: Plan Nacional sobre Drogas.

Botvin, Gilbert J. (1995). Entrenamiento en habilidades para la vida y prevención del consumo de drogas en adolescentes: consideraciones teóricas y hallazgos empíricos. Psicología Conductual, 3 (3), 333-356.

Bueno, Francisco J. (2009). Nuevas Tecnologías: factores de protección y de riesgo. XVIII Jornada sobre drogodependencias. SMS: Ilegan las adicciones a las nuevas tecnologías. 3 de diciembre 2009. Valencia: Plan Municipal de Drogodependencias. Ayuntamiento de Valencia.

Burkhart, G. (2011). Environmental drug prevention in the EU. Why is it so unpopular?. Adicciones, Vol. 23 (2). 87-100.

Burkhart, G. (2013). North American drug prevention programmes: are they feasible in European cultures and contexts? Lisbon: EM- CDDA. doi:10.2810/41791

Chavez, E.L., Oetting, R.R. y Swaim, R.C. (1994). Dropout and delinquency: Mexican American and Caucasian Non-Hispanic youth. Journal of Clinical Child Psychology, 23, 47-55.

Centre de Prevencio i Intervencio en drogodependencies, (2013). Guía para la elaboración de un plan local de prevención de drogodependencias. Barcelona. Diputación Barcelona.

Clayton, R.R. (1992). Transitions in drug use: Risk and protective factors». En M. T. Laespada, I. Iraurgi y E. Aróstegi (Eds). (2004). Factores de Riesgo y de Protección frente al Consumo de Drogas: Hacia un Modelo Explicativo del Consumo de Drogas en Jóvenes de la CAPV. Bilbao. Instituto Deusto de Drogodependencias (Universidad de Deusto).

Concilio Vaticano II. Gravissimum Educationis. Recuperado el 24 de abril del 2017 dhttp://www.vatican.va/archive/hist_councils/ii_vatican_council/documents/vat-ii_decl_19651028_gravissimum-educationis_sp.html 
Glantz y R. Pickens (1996). Vulnerability to drug abuse. Washington, DC: American Psychological Association.

Cyrulnik, B. (2002). Los patitos feos. La resiliencia: una infancia infeliz no determina la vida. Barcelona: Gedisa.

FAD, (2011). Guía Didáctica: Construye tu Mundo. Madrid. Fundación Ayuda contra la Drogadicción.

Félix-Ortiz, M. y Newcomb, M.D. (1999). Vulnerability for drug use among latino adolescents. Journal of Community Psychology, 27, 257-280.

Ferguson, D.M. y Lynskey, M.T. (1996). Adolescent resilience to family adversity. Journal of Child Psychology and Psychiatry, 37, 281-292.

García, F.; Portillo, J.; Romo, J. y Benito, M. (2007). Nativos digitales y modelos de aprendizaje. IV Simposio Pluridisciplinar sobre Diseño, Evaluación y Desarrollo de contenidos educativos reutilizables. 19, 20 y 21 Septiembre de 2007. Bilbao: Universidad del País Vasco.

Garmezy, N. (1991). Resiliency and vulnerability to adverse developmental outcomes associated with poverty. American Behavioral Scientist, 31, 416-430.

Gordon, Robert. (1987). An operational classification of disease prevention. En Jane A. Steinberg y Morton M. Silverman (Eds.), Preventing Mental Disorders (pp. 20-26). Rockville, MD: U.S. Department of Health and Human Services.

Hawkins, J.D., Catalano, R.f. y Miller, J.Y. (1992). Risk and protective factors for alcohol and other drug problems in adolescence and early adulthood: Implications for substance abuse prevention. Psychological Bulletin, 112, 64-105.

Hernandez Sanchez, M., Pastor Munoz, P. Y Gil Fernandez, N. (2016). Beneficios del ejercicio físico para el toxicomano. $V$ Congreso Internacional de deporte inclusivo, libro de actas. Universidad de Almeria. 25 al 29 de mayo de 2015.

INE, (2016). Equipamiento y uso de TIC en los hogares, Año 2016. Madrid: Instituto Nacional de Estadística.

Recuperado de:

http://www.ine.es/dyngs/INEbase/es/operacion.htm?c=Estadistica_C\&cid=1254736176741\&menu= ultiDatos\&idp $=1254735576692$

INTECO, (2009). Guía legal sobre ciberbullying y grooming. Instituto Nacional de Tecnologías de la Comunicación. Pamplona: Navarra.

Recuperado de:

http://www.inteco.es/Seguridad/Observatorio/guias/guiaManual_groming_ciberbullying

Jessor, R., Donovan, J. E. y Costa, F. M. (1991). Beyond adolescence: Problem behavior and young adult development. Cambridge: Cambridge University Press.

KiVa School, (2017). Programa Kiva contra acoso escolar: Turku. Finlandia.

Recuperado de: http://www.kivaprogram.net/spain

Kumpfer, K.L., Szapocznik, J., Catalano, R., Clayton, R.R., Liddle, H.A., Mcmahon, R., Millman, J., Orrego, M.E.V., Rinehart, N., Smith, I., Spoth, R. y Steele, M. (1998). Preventing substance abuse among children and adolescents: Familycenteredapproaches. Rockville, MD: Department of Health and Human Services, Substance Abuse and Mental Health Services Administration, Center for Substance Abuse Prevention.

Moncada, S. (1997). Factores de riesgo y de protección en el consumo de drogas. En Plan Nacional sobre Drogas (Ed.), Prevención de las drogodependencias. Análisis y propuestas de actuación (pp. 85-101). Madrid: Plan Nacional sobre Drogas.

OMS, (2002). Informe Mundial sobre la violencia y la salud. Ginebra: Organización Panamericana de la Salud. Organización Mundial de la Salud. 
Pérez Vallejo, A.M. y Pérez Ferrer, F. (2016). Bullying, ciberbullying y acoso con elementos sexuales: desde la prevención a la reparación del daño. Madrid: Dykinson, S.L.

Perry, C.L. y Kelder, S.H. (1992). Models for effective prevention. Journal of Adolescent, 13, 355363.

Petterson, P.L., Hawkins, J.D. y Catalano, R.F. (1992). Evaluating comprensive community drug risk reduction interventions. Design challenges and $r$ ecommendations. Evaluation Review, 16, 579-602.

Pollard, J.A., Catalano, R.F., Hawkins, J.D. y Arthur, M. W. (1997). Development of a school-based survey measuring risk and protective factors predictive of substance abuse, delinquency, and other problem behaviors in adolescent population. Manuscrito no publicado.

Prensky, Marc (2001). Digital natives, digital immigrants. On the Horizon, 9 (6), 1-6.

Rutter, M. (1985). Resilience in the face of adversity: Protective factors and resistance

to psychiatric disorders. British Journal of Psychiatry, 147, 598-611.

Salazar, José y Bustos, Lorena (2002). Prevención integral en drogodependencias. Revista Novedades Educativas, 133, año 13, 32-35.

Salazar Ascencio, J. I y Williamson Castro, G. (2015). Modelo integral de intervención para la prevención en drogodependencias. Aportes para la pedagogía social. Perfiles Educativos, Suplemento 2015. XXXVII(148), pp. 20-27.

Tevez, H. (2006). La ética como prevención. Ideas para pensar entre padres y adolescentes. En Fernandez D’Adam y Federico Ré. (Coords.). Resilencia, ética y prevención. (pp. 143-156). Buenos Aires: Gabas Editorial.

Turbi, Ángel M. y López, Roque (2010). Encuesta sobre uso de las TIC en población escolar, resultados preliminares. No publicado.

Turbi, Angel M. (2013). Violencia en las Redes Sociales: Prevención y Tratamiento. En Magdalena Jimenez y Francisco J Del Pozo. (Coords.). Propuestas Didácticas para la Igualdad (pp. 121145) Granada: Editorial Natívola.

Turbi, Angel M. (2017). El entorno de las conductas adictivas. Blog de psicología. Valencia: Universidad Católica de Valencia "San Vicente Mártir".

Recuperado de: http://blogs.ucv.es/postgradopsocologia/2017/07/15/el-entorno-de-las-conductasadictivas-ha-sido-y-es-en-la-actualidad-un-espacio-que-conlleva-su-complejidad/

Valmaggia, L.R., Day, F.L., Kroll, J., Laing, J., Byrne, M., Fusar-Poli, P. Y McGuire, P. (2015). Bullying victimisation and paranoid ideation in people al ultra high risk for psychosis. Schizophrenia Research, 168 (68-73). 